\section{ORIGINAL RESEARCH}

Z. Kulcsár

E. Houdart

A. Bonafé

G. Parker

J. Millar

A.J.P. Goddard

S. Renowden

G. Gál

B. Turowski

K. Mitchell

F. Gray

M. Rodriguez

R. van den Berg

A. Gruber

H. Desal

I. Wanke

D.A. Rüfenacht

\title{
Intra-Aneurysmal Thrombosis as a Possible \\ Cause of Delayed Aneurysm Rupture after Flow-Diversion Treatment
}

BACKGROUND AND PURPOSE: FD technology enables reconstructive repair of otherwise difficult-totreat intracranial aneurysms. These stentlike devices may induce progressive aneurysm thrombosis without additional implants and may initiate complete reverse vessel remodeling. The associated vascular biologic processes are as yet only partially understood.

MATERIALS AND METHODS: From 12 different centers, 13 cases of delayed postprocedural aneurysm rupture were recorded and analyzed. Symptom, aneurysm location and morphology, and the time elapsed from treatment until rupture were analyzed.

RESULTS: There were 10 internal carotid and 3 basilar artery aneurysms. Mean aneurysm diameter was $22 \pm 6 \mathrm{~mm}$. Eleven patients were symptomatic before treatment. A single FD was used for all saccular aneurysms, while fusiform lesions were treated by using multiple devices. A supplementary loose coiling of the aneurysm was performed in 1 patient only. Ten patients developed early aneurysm rupture after FD treatment (mean, 16 days; range, 2-48 days); in 3 patients, rupture occurred 3-5 months after treatment. In all cases, most of the aneurysm cavity was thrombosed before rupture. The biologic mechanisms predisposing to rupture under these conditions are reviewed and discussed

CONCLUSIONS: FDs alone may modify hemodynamics in ways that induce extensive aneurysm thrombosis. Under specific conditions, however, instead of reverse remodeling and cicatrization, aggressive thrombus-associated autolysis of the aneurysm wall may result in delayed rupture.

ABBREVIATIONS: $A A A=$ abdominal aortic aneurysm; approx. = approximate; $A R=$ aspect ratio; $\mathrm{CCF}=$ carotid cavernous fistula; $\mathrm{DSA}=$ digital subtraction angiography; $\mathrm{FD}=$ flow diverter; $\mathrm{HE}=$ hematoxylin-eosin; ICA = internal carotid artery; $L=$ left; Max. = maximum; $N A=$ not applicable; $\mathrm{PAO}=$ parent artery occlusion; $\mathrm{R}=$ right

$\mathbf{T}$ he advent of FD implants has provided a new endovascular tool for reconstructive treatment and vascular remodeling of broad-based, large or giant, and fusiform aneurysms, for

Received August 10, 2010; accepted after revision September 16.

From the Department of Neuroradiology (Z.K., I.W., D.A.R.), Swiss Neuro Institute Hirslanden, Clinic Hirslanden, Zurich, Switzerland; Department of Diagnostic and Interventional Neuroradiology (E.H.) and Department of Pathology (F.G.), Hopital Lariboisiere, Paris, France; Department of Neuroradiology (A.B.), University Hospital Center, Gui de Chauliac Hospital, Montpellier, France; Department of Neuroradiology (G.P.), Royal Prince Alfred Hospital, Sydney, Australia; Department of Neuroradiology (J.M.), Wessex Neurological Centre, Southampton General Hospital, Southampton, England; Department of Neuroradiology (A.J.P.G.), Leeds General Infirmary, Leeds, United Kingdom; Department of Neuroradiology (S.R.), Frenchay Hospital Bristol, Bristol, United Kingdom; Department of Neuroradiology (G.G.), Odense University Hospital, Odense, Denmark; Institute of Radiology/ Neuroradiology (B.T.), University of Dusseldorf, Dusseldorf, Germany; Department of Medical Imaging (K.M.), Royal Brisbane and Women's Hospital, Herston, Queensland, Australia; Department of Forensic Medicine (M.R.), Sydney Southwest Area Health Service, Sydney, Australia; Department of Radiology (R.v.d.B.), Acamedic Medical Center, Amsterdam, the Netherlands; Department of Neurosurgery (A.G.), Medical University Vienna, Vienna General Hospital, Vienna, Austria; and Department of Neuroradiology (H.D.), University Hospital Laënnec Hospital, CHU de Nantes, France.

The large number of authors is justified by the fact that altogether 13 centers participated in the research with 15 neurointerventional specialists and 2 neurohistopathologists. All these authors contributed to the work

I. Wanke, Z. Kulcsár, G. Gal, and A.J.P. Goddard are contractual proctors for SILK FD implantation.

Please address correspondence to Zsolt Kulcsár, MD, Neuroradiology, Swiss Neuro Institute, Kliník Hirslanden, Witellikerstr 40, 8032 Zurich, Switzerland; e-mail: zsolt.kulcsar@ hirslanden.ch

Indicates open access to non-subscribers at www.ajnr.org

Indicates article with supplemental on-line table.

DOI 10.3174/ajnr.A2370 which conventional reconstructive surgical or endovascular treatment methods are either not feasible or are prone to a high recurrence rate. ${ }^{1,2}$ Although long-term follow-up data are not yet available, promising experience is accumulating for treatment of such aneurysms. ${ }^{1-5}$ On the basis of flow-diversion concepts alone, progressive aneurysm thrombosis and reverse remodeling of the aneurysm and the vessel wall are expected, without the use of additional embolic material. The time course of this healing process is as yet unclear, and there likely exist individual differences, influenced by factors including the type of FD and resultant flow change, parent vessel geometry, aneurysm size and morphology, and blood coagulation parameters.

After the first reports of aneurysms rupturing weeks after flow-diversion treatment with the Silk FD (Balt Extrusion, Montmorency, France $)^{6}$ and the competing Pipeline Embolization Device (ev3, Plymouth, Minnesota) (P.K. Nelson, unpublished data, Annual Meeting of the American Society of Neuroradiology, Boston, Massachusetts; May 19, 2010), our aim was to collect similar cases, analyze them, and try to understand the mechanisms leading to delayed rupture during the healing process.

\section{Materials and Methods}

This retrospective analysis describes 13 cases from 12 centers worldwide of delayed hemorrhage following implantation of the Silk FD device (Balt Extrusion). These cases represent all complications of this type from the 12 centers, occurring in the 16 months before March 2010. One case (No. 7) has been previously published as a case report. ${ }^{6}$ According to the internal records of the company, through the 
end of March 2010, >1500 FD procedures were performed with the device worldwide. Given the lack of reliable worldwide registry on procedures and complications with the device, the exact incidence of delayed ruptures could not be assessed.

In all cases, treatment was performed with the intention to cure a large or giant wide-neck saccular or fusiform intracranial aneurysm otherwise difficult to treat, and flow diversion was judged to be the most appropriate treatment option (On-line Table). All patients received aspirin and clopidogrel therapy before treatment, which was continued for 2-3 months after FD implantation (depending on the individual center) or until rupture. Eight of the 9 saccular lesions were treated with the FD alone. One saccular aneurysm was treated with the FD and additional very loose coil packing of the aneurysm. The fusiform aneurysms $(n=4)$ were treated with initial implantation of 1 or 2 LEO + stents (Balt) to provide intravascular support for the complementary added less rigid FD. All patients underwent follow-up imaging by CT/CT angiography or MR/MR angiography between treatment and delayed aneurysm rupture; all, except 1 , underwent imaging after rupture.

To identify common features in these cases, we analyzed risk factors for rupture and symptoms before and after FD treatment, aneurysm morphology and AR (dome height to neck ratio), treatment details, clinical evolution, and time between treatment and rupture. Changes in flow pattern (ie, inflow jet, contrast material stagnation) before and after FD implantation were assessed by reviewing the relevant DSA sequences (2-3 images/s); computational fluid dynamic analyses were not performed. Evolution and volume of aneurysm thrombosis after FD treatment were estimated from the available brain imaging studies. Pathologic samples, when available, were also assessed.

\section{Results}

\section{Patient and Aneurysm Characteristics}

The mean age of the 13 patients was $60 \pm 11$ years, and there were 9 women. Eleven aneurysms were symptomatic, with neurologic symptoms due to mass effect or recent onset of alarming headaches. Nine patients had known hypertension; 2 were active smokers. Two patients were admitted with a suspected sentinel leak and were treated in the acute phase.

The aneurysms arose from the cavernous $(n=2)$ and supraclinoid $(n=8)$ ICAs and the basilar trunk $(n=3)$. Of the 4 fusiform aneurysms, 3 affected the basilar trunk and 1, the terminal ICA. All 9 saccular lesions were broad-based, involving a major part of the circumference of the parent vessel. All aneurysms were large or giant, with a mean diameter of $22 \pm 6$ $\mathrm{mm}$. For the saccular aneurysms, the mean dome size was $20 \pm 3 \mathrm{~mm}$ and the mean $\mathrm{AR}$ was $3.1 \pm 0.9$. The mean \pm 7.5 SD transverse diameter of the fusiform aneurysms was $27 \mathrm{~mm}$ (range, 17-35 mm). Two of the saccular aneurysms showed partial thrombosis (approximately $70 \%$ and $20 \%$ of the aneurysm volume) before treatment. PAO was not considered as an alternative treatment option in 9 of the 13 cases. In the other 4 cases, a test occlusion was performed; in only 1 was PAO considered feasible.

\section{Treatment}

At the end of the procedure, the FD was implanted as desired in 11 of 13 cases. In 2 cases, the device was not completely open despite postdeployment manipulations. Thromboembolic complications occurred in 4 patients during the procedure, resulting in ischemia-related neurologic deficits.

\section{Flow-Pattern Changes after FD Implantation}

On the basis of conventional DSA images, an inflow jet was identified before device implantation in 12 of 13 patients. After device implantation, this was still present and clearly identifiable in 11 cases (Fig 1). FD placement increased contrast material stagnation in the aneurysm cavity in all cases.

\section{Clinical and Radiologic Evolution Following FD Implantation}

From the 13 patients, 10 had an acute and diffuse subarachnoid hemorrhage and 2 patients with a cavernous aneurysm developed a CCF, all suggestive of rupture of the treated aneurysm. One patient (No. 1) with a 21-mm proximal M1 segment aneurysm had a hemorrhage with intraventricular extension at the head of the caudate nucleus, which was in close conjunction with the dome of the aneurysm. This bleeding was also suggestive of aneurysm rupture rather than of hemorrhagic transformation of an infarct because no ischemic changes were seen on the 8-hour posttreatment control CT scan. Four patients developed severe headaches during the period between treatment and rupture. Eight patients received corticosteroids at some time after treatment for aneurysm mass effect-reduction purposes. On the basis of the time between treatment and aneurysm rupture, patients were separated into groups with early $(<3$ months) and late (3-5 months) rupture. Patients in the early rupture group $(n=10)$ were still receiving both aspirin and clopidogrel treatment. The mean treatment-to-rupture time was 16 days (range, $2-48)$. Patients in the late rupture group $(n=3)$ were receiving aspirin alone. The mean treatment-to-rupture time was 132 days (range, 110-150 days). Most interesting, 1 carotid cavernous aneurysm (No.11) still showed complete patency at 3 months, at which time clopidogrel treatment was stopped. The aneurysm ruptured 20 days later, at which time it was already largely thrombosed.

\section{Aneurysm Thrombosis}

At follow-up imaging, 12 of the aneurysms were partially or completely thrombosed. In the case in which additional coil packing was performed, thrombosis could not be assessed. Two aneurysms were completely thrombosed, and in the other cases, most of the lumen was occluded by thrombus, as estimated from the imaging studies (mean occlusion, 80\%; On-line Table).

\section{Pathology Results}

Postmortem dissection and detailed histopathologic examination were performed in patients 5 and 6 , showing a massive subarachnoid hemorrhage. Macroscopically, both aneurysms showed massive organizing intraluminal thrombus and mural thinning at the site of rupture (Fig 2). Microscopically, the aneurysm wall at the site of the rupture was extremely thin, with mural necrosis, loss of fibrous tissue and medial smooth muscle cells, and infiltration by macrophages extending from the adventitial surface (Fig 3). 

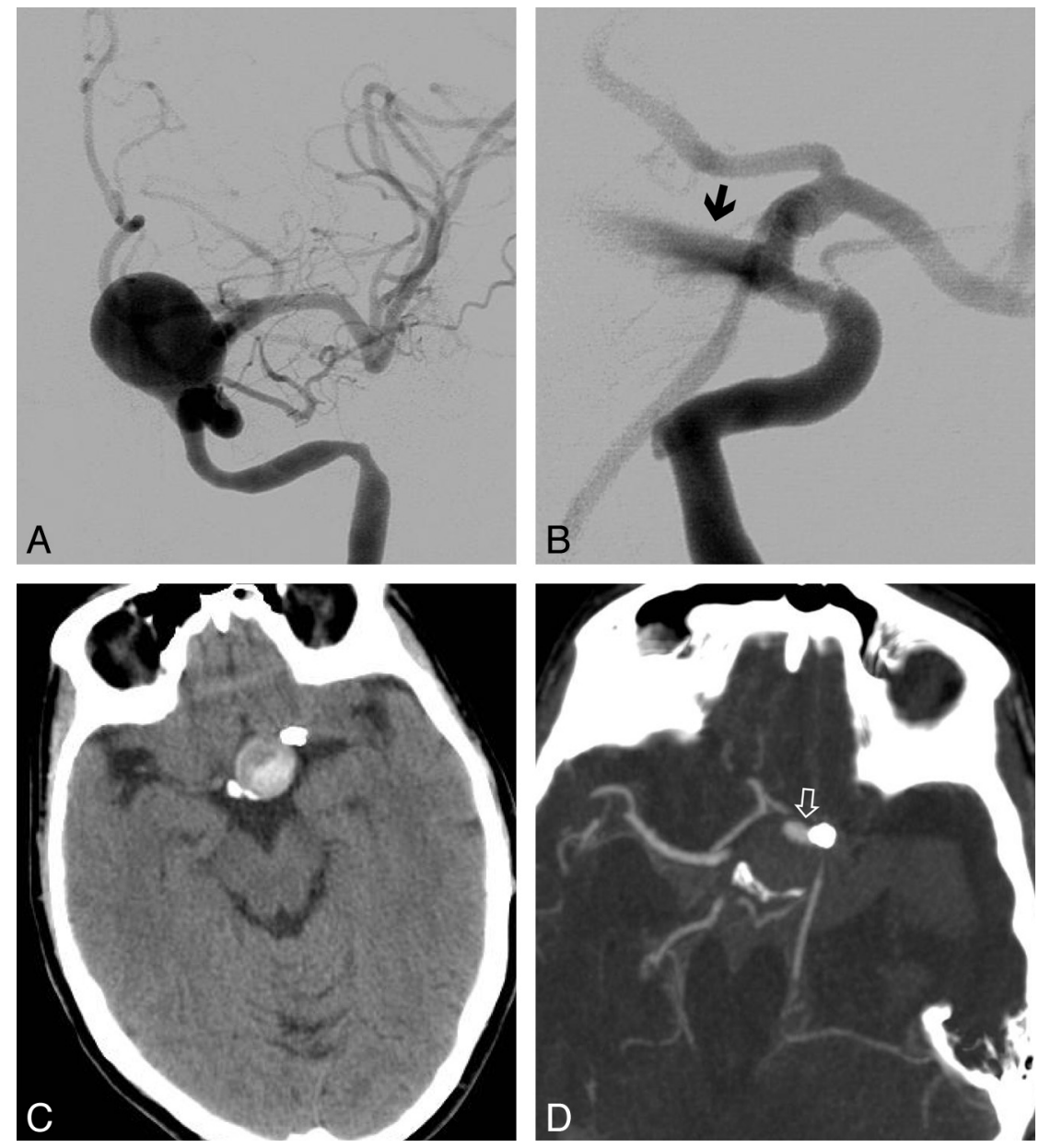

Fig 1. Patient No. 3. A, Posteroanterior view DSA image of an incidentally discovered left ICA parophthalmic aneurysm. B, Persistence of the inertia-driven inflow jet (arrow) is shown after device deployment. $C$ and $D$, Nonenhanced CT scan 2 days after intervention shows already partial thrombosis of the aneurysm (C), and a CT angiogram at day 5 shows massive subarachnoid hemorrhage and only a minor portion of the aneurysm that is still perfused, corresponding to the inflow jet area (open arrow) (D).

\section{Discussion}

In this multicenter case series of aneurysms rupturing after flow-diversion treatment, we analyzed each case and identified common features potentially contributing to delayed degradation of the aneurysm wall and subsequent rupture.

\section{Rupture-Risk Assessment before Treatment}

All patients belonged to a relatively high-risk group, most presenting with a combination of multiple risk factors for rupture. Eleven aneurysms were symptomatic before treatment, and symptomatic aneurysms have a relative risk for rupture of 8.3 compared with asymptomatic ones. ${ }^{7}$ Most patients had hypertension. With a mean aneurysm size of $22 \mathrm{~mm}$ and with all lesions being $>15 \mathrm{~mm}$, the rupture risk further increased. ${ }^{8}$

For saccular aneurysms, a high AR $(>1.6)$ has been shown to correlate with aneurysm rupture. ${ }^{9}$ In our series, the mean AR for the 9 saccular aneurysms was 3.1 and was $\geq 1.8$ in all cases. In a study by Ujiie et al, ${ }^{10}$ an AR of $>1.6$ correlated with slow flow in the dome area, resulting in low wall shear stress. This is known to cause aggregation of blood cells along the intimal surface, ${ }^{10}$ endothelial dysfunction, and cell loss. ${ }^{11}$ This result, in turn, leads to intimal damage, thrombosis, and intramural inflammation with further degeneration of the aneurysm wall. ${ }^{12}$ In principle, these processes, leading to degeneration of the aneurysm wall and eventual rupture, may also have important biologic effects on the aneurysm wall after treatment. Although most large and giant aneurysms, like in our series, tend to have a high AR, this indirect sign of aneurysm wall vulnerability and fragility may become more relevant when addressing the delayed rupture risk of aneurysms smaller than those from this series.

\section{Treatment Effects with FD Implantation}

FD implantation resulted in significant flow changes in all cases; however, inflow jets as recorded before treatment were still present immediately after treatment in most patients. With in vitro studies and numeric simulations of FD hemodynamic effects, such a lack of flow change has been observed with inertia-driven flow (when the arterial flow points to inside the aneurysmal sac) compared with shear-driven flow. ${ }^{13}$ Therefore, FD effects in an individual patient depend on local flow conditions and are mainly governed by parent vessel geometry and aneurysm configuration. A possible undesirable effect of insufficient flow diversion may be the induction of a detrimental intra-aneurysmal flow pattern. FD treatment could therefore lead to a sudden change in flow pattern, with consequent increased stress to areas that were not previously exposed to strain. This might play a leading role in rupture if there is no or only partial thrombosis.

Treatment with FDs changed the intra-aneurysmal hemo- 

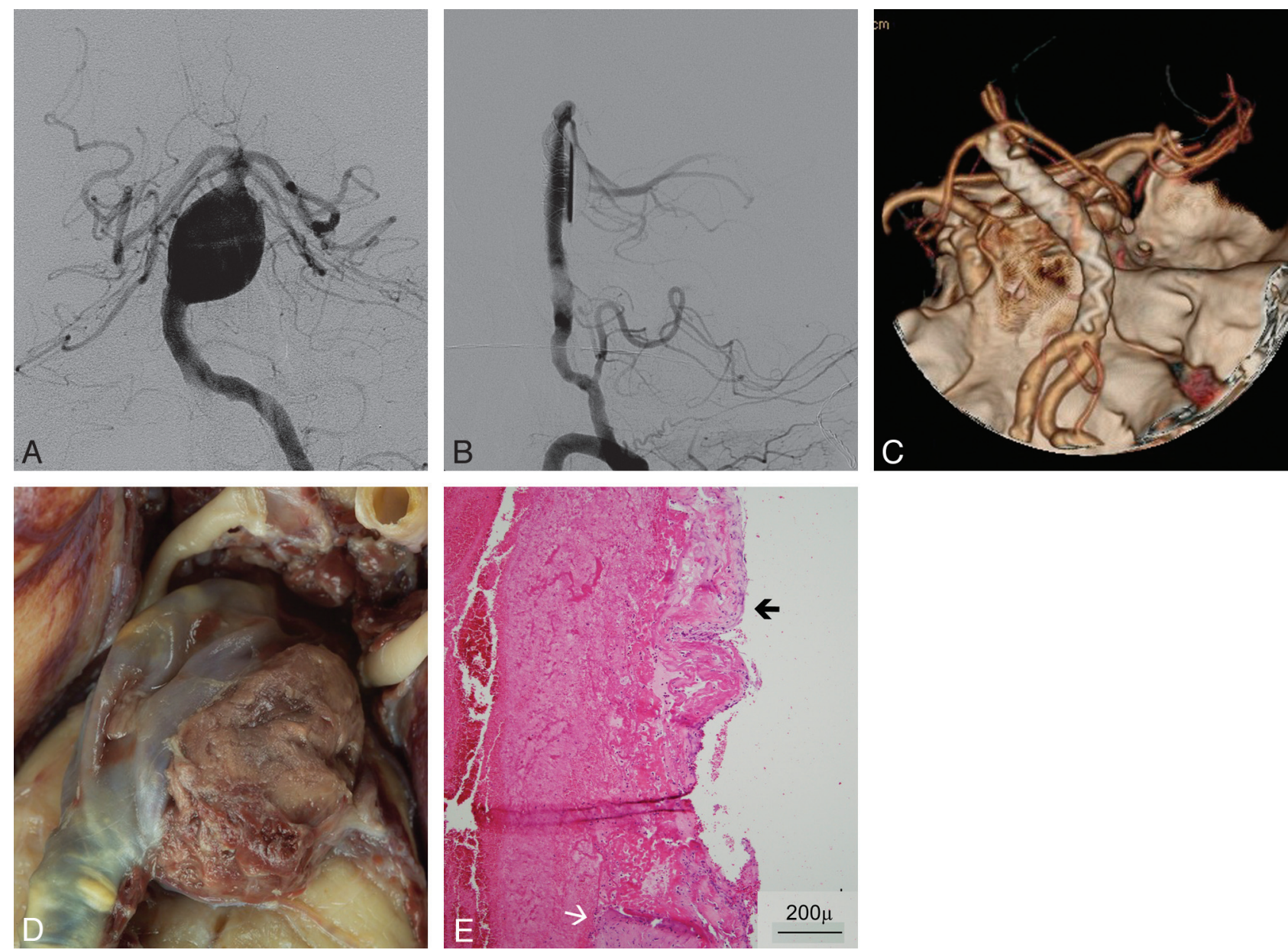

Fig 2. Patient No. 6. $A$ and $B$, Posteroanterior view DSA image of a symptomatic fusiform aneurysm of the basilar trunk $(A)$ and lateral view after FD treatment $(B)$ demonstrate contrast stasis in the aneurysm. C, 3D CT angiogram obtained 3 days after treatment shows patency of the basilar trunk and lack of aneurysm filling. $D$, Macroscopic view of the basilar artery

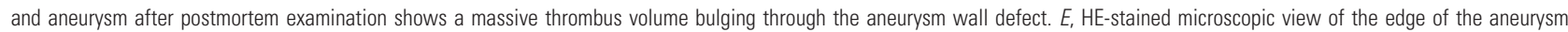
wall rupture shows a partially maintained but vanishing adventitia (black arrow) and disappearance of the vascular tunica media (white arrow).

dynamics resulting, in all cases, in complete or significant thrombus formation in the aneurysm cavity. The reduced but persisting intra-aneurysmal flow could provide a basis for continuous thrombus renewal. This is known to favor autolytic aggressive thrombus formation. ${ }^{14}$

\section{Role of Intra-Aneurysmal Thrombus in Delayed Aneurysm Rupture}

Thrombus developed in the aneurysm sac in all cases after FD treatment, suggesting that in some circumstances, intraluminal thrombus may contribute to the pathophysiologic cascade ending in rupture in both untreated and treated aneurysms. In clinical studies, besides increased de-endothelialization and aneurysm wall infiltration by leukocytes, ${ }^{15,16}$ the presence of fresh and organizing luminal thrombus was identified as a key factor leading to aneurysm wall degradation and correlating with aneurysm rupture. ${ }^{15}$

On the other hand, thrombus formation is the first step leading to permanent aneurysm repair through cicatrization and reverse remodeling of the vessel wall. Coil and FD treatment have demonstrated well their great potential to induce this pathway of cure through thrombosis. ${ }^{1,2}$ It is unclear why thrombosis may lead to cure in some cases, whereas in others, it appears to trigger increased autolysis, which may overload the biologic defense mechanisms of the vessel wall and result in aneurysm rupture.

Similar pathologic processes are observed and have been extensively investigated in AAAs. In AAAs, mural thrombus forms along the aneurysm wall in permanent contact with the flowing blood and undergoes continuous thrombolysis and renewal. ${ }^{14}$ Mural thrombi within AAAs are characterized by an absence of organization and cell colonization ${ }^{17}$ and are associated with a thinner arterial wall, more extensive elastolysis, a lower attenuation of smooth muscle cells in the media, and a higher level of immunoinflammation in the adventitia. ${ }^{14}$ By aggregating platelets continuously entrapping circulating white blood cells and absorbing plasma components, the mural thrombus acts as a source of secreted proteases within the aneurysm, leading to further chemical degradation and weakening of the aneurysm wall with subsequent evolution of the aneurysm. ${ }^{17}$ The volume of mural thrombus correlates with protease activity both in the thrombus itself and within the aneurysm wall, ${ }^{18}$ suggesting that the bigger the thrombus, the more important its biochemical effects.

It is tempting to apply these observations to intracranial aneurysms. All the aneurysms in our series contained a significant volume of thrombus following FD treatment, which filled most or the entire volume of the aneurysm cavity. In the 

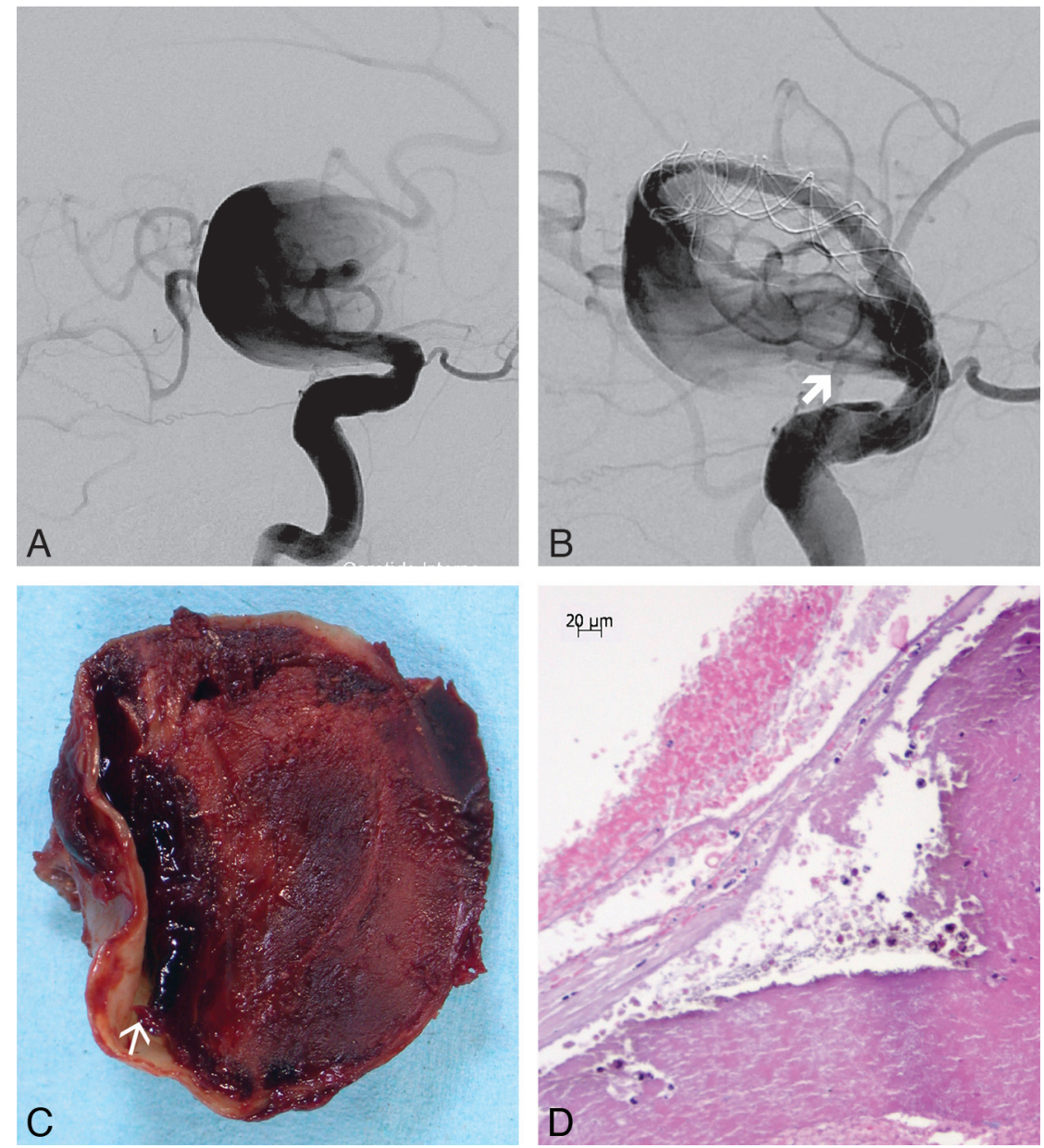

Fig 3. Patient No. 5. $A$ and $B$, Giant fusiform aneurysm of the right distal ICA, right oblique view before $(A)$ and after FD implantation, with a persisting inflow jet (thick arrow) ( $B$ ). $C$, Cross-section of the aneurysm after postmortem examination shows organized thrombus filling most the aneurysm lumen. Note a dissecting cavity with liquefied clot between the organized thrombus and the thick part of the aneurysm wall (arrow). D, Microscopic view of the aneurysm after HE-staining at the rupture site shows necrosis and almost complete disappearance of the aneurysm wall.

2 cases examined histopathologically, thrombus without significant organization or cellular colonization was identified, as described in AAA. That thrombosis may precipitate rupture was also suggested in patient 11, in whom, as described above, delayed rupture occurred only after thrombus developed.

In contrast, clinical observations of aneurysms following spontaneous partial thrombosis do not suggest that thrombosis significantly increases the risk of rupture. ${ }^{19}$ However the biologic consequences of stepwise thrombosis may not be the same as those for a large thrombus developing rapidly. One could suggest that depending on the quality, quantity, and evolution of the thrombus, the aneurysm wall might be exposed to varying thrombus-induced autolytic activities, though this possibility is hypothetic. Furthermore, in the presence of a large thrombus, defense mechanisms opposing autolysis and wall degradation may be overwhelmed by a large enzymatic overload. Whether cicatrization and reverse remodeling or rupture occur may depend on the balance between pro- and antiautolytic activities in the aneurysm wall. Our observations support this hypothesis because the ruptured aneurysms in our series were large and thin-walled and contained extensive thrombus that developed during a short period.

The temporary swelling and increased volume of a throm- bosed aneurysm following FD treatment or $\mathrm{PAO}^{20}$ might also be explained by a similar mechanism, though following gradual rather than rapid thrombosis (the latter favoring rupture).

Cicatrization and reverse remodeling with a decrease in aneurysm volume are thought to start only after complete thrombosis. ${ }^{21}$ According to our hypothesis, by promoting thrombus within the aneurysm sac and by isolating it from the circulation, FD devices facilitate this process. Cicatrization and reverse remodeling are favored when thrombus in the aneurysmal cavity stops the continuous process of renewal, an event that becomes more likely following sealing of the luminal surface of the thrombus by a layer of neointimal tissue and in-growth of mesenchymal cells.

\section{Major Common Features of the Ruptured Aneurysms}

The main goal of this analysis was to suggest a mechanism to explain the observed delayed aneurysm wall degradation and rupture. We have identified 4 features in our series that may be associated with an increased risk of delayed rupture:

1) Large and giant aneurysms, potentially able to contain large rapidly accumulated thrombi

2) Symptomatic aneurysms suggesting recent growth and wall instability 
3) Saccular aneurysms with an AR of $>1.6$

4) Morphologic characteristics predisposing to an inertiadriven inflow, not necessarily addressed by a FD device.

In this series, at least 3 of the 4 features were present in all patients, and in 7 patients, all 4 features were present. Needless to say, our study was not designed and does not enable us to assess the relative risk of delayed rupture in the presence of $\geq 1$ of these features. Nevertheless, delayed-rupture events for all aneurysms with the above-mentioned characteristics remain rare after this form of treatment; however, to date, no scientifically reliable registry data are available to address the exact incidence. We suggest that for delayed rupture to occur, a complex interaction of multiple factors is required. For intracranial aneurysms, these factors and interactions have, as yet, been only partially unraveled.

\section{Proposed Treatment Strategy to Avoid Ruptures}

On the basis of our observations and our hypothesis, we suggest that measures to prevent the buildup of unstable thrombus exhibiting high and aggressive autolytic characteristics following flow diversion may reduce the incidence of delayed rupture. This might be achieved with aneurysm filling, with the addition of coils, or by increasing the flow-diverting effect of the implants. Coils may exhibit favorable effects in stabilizing thrombus, different from stagnation through flow diversion alone. Deconstructive methods like PAO remain a very valuable and safe solution when hemodynamically tolerated. We should not forget the high natural bleeding risk in this specific group of patients with large and giant aneurysms and should balance the treatment risk accordingly.

The major limitations of this study are the retrospective nature of the analysis, the lack of exact data on the incidence of aneurysm ruptures after FD treatment, and the lack of a control group that would make comparison with other similar aneurysms not rupturing after FD treatment possible.

\section{Conclusions}

Intra-aneurysmal thrombus formation after flow-diversion treatment of large and giant intracranial aneurysms may trigger a complex cascade of biologic events, known to have deleterious effects on the aneurysm wall. Thrombus formation is a recognized necessary step to cure aneurysms by endovascular methods by leading, in most cases, to reverse remodeling and cicatrization. Under specific conditions, however, aggressive autolytic effects of the thrombus may lead to delayed rupture. The effectiveness and, therefore, the success of this otherwise very promising technique may be improved by identifying aneurysms at risk of delayed rupture and by using strategies to avoid the development of unstable clot.

\section{Acknowledgments}

We are grateful to Balt (Montmorency, France), the manufacturer of SILK FD devices, for facilitating the identification of cases of serious adverse events due to hemorrhage related to the use of the SILK FD device. This fostered international collaboration among colleagues, leading to a better understanding of this type of serious complication. We also acknowledge the contribution of Paolo Machi, MD, to this work.

\section{References}

1. Lylyk P, Miranda C, Ceratto R, et al. Curative endovascular reconstruction of cerebral aneurysms with the Pipeline embolization device: the Buenos Aires experience. Neurosurgery 2009;64:632-42, discussion 642-43, quiz N6

2. Szikora I, Berentei Z, Kulcsar Z, et al. Treatment of intracranial aneurysms by functional reconstruction of the parent artery: the Budapest experience with the Pipeline embolization device. AJNR Am J Neuroradiol 2010;31:1139-47. Epub 2010 Feb 11

3. Fiorella D, Woo HH, Albuquerque FC, et al. Definitive reconstruction of circumferential, fusiform intracranial aneurysms with the Pipeline embolization device. Neurosurgery 2008;62:1115-20, discussion 1120-21

4. Jahromi BS, Mocco J, Bang JA, et al. Clinical and angiographic outcome after endovascular management of giant intracranial aneurysms. Neurosurgery 2008;63:662-74, discussion 674-75

5. Sluzewski M, Menovsky T, van Rooij WJ, et al. Coiling of very large or giant cerebral aneurysms: long-term clinical and serial angiographic results. $A J N R$ Am J Neuroradiol 2003;24:257-62

6. Turowski B, Macht S, Kulcsar Z, et al. Early fatal hemorrhage after endovascular cerebral aneurysm treatment with a flow diverter (Silk-Stent): do we need to rethink our concepts? Neuroradiology 2010 Mar 26 [Epub ahead of print]

7. Rinkel GJ, Djibuti M, Algra A, et al. Prevalence and risk of rupture of intracranial aneurysms: a systematic review. Stroke 1998;29:251-56

8. Wiebers DO, Whisnant JP, Huston J 3rd, et al, for the International Study of Unruptured Intracranial Aneurysms Investigators. Unruptured intracranial aneurysms: natural history, clinical outcome, and risks of surgical and endovascular treatment. Lancet 2003;362:103-10

9. Ujiie $\mathrm{H}$, Tamano $\mathrm{Y}$, Sasaki $\mathrm{K}$, et al. Is the aspect ratio a reliable index for predicting the rupture of a saccular aneurysm? Neurosurgery 2001;48:495-502, discussion 502-03

10. Ujiie H, Tachibana H, Hiramatsu O, et al. Effects of size and shape (aspect ratio) on the hemodynamics of saccular aneurysms: a possible index for surgical treatment of intracranial aneurysms. Neurosurgery 1999;45:119-29, discussion 129-30

11. Malek AM, Alper SL, Izumo S. Hemodynamic shear stress and its role in atherosclerosis. JAMA 1999;282:2035-42

12. Tulamo R, Frösen J, Hernesniemi J, et al. Inflammatory changes in the aneurysm wall: a review. J Neurointervent Surg 2010;2:120-30

13. Augsburger L, Farhat M, Reymond P, et al. Effect of flow diverter porosity on intraaneurysmal blood flow. Klin Neuroradiol 2009;19:204-14

14. Touat Z, Ollivier V, Dai J, et al. Renewal of mural thrombus releases plasma markers and is involved in aortic abdominal aneurysm evolution. Am J Pathol 2006;168:1022-30

15. Frosen J, Piippo A, Paetau A, et al. Remodeling of saccular cerebral artery aneurysm wall is associated with rupture: histological analysis of 24 unruptured and 42 ruptured cases. Stroke 2004;35:2287-93

16. Kataoka K, Taneda M, Asai T, et al. Structural fragility and inflammatory response of ruptured cerebral aneurysms: a comparative study between ruptured and unruptured cerebral aneurysms. Stroke 1999;30:1396-401

17. Fontaine V, Jacob MP, Houard X, et al. Involvement of the mural thrombus as a site of protease release and activation in human aortic aneurysms. $A m J$ Pathol 2002;161:1701-10

18. Coutard M, Touat Z, Houard X, et al. Thrombus versus wall biological activities in experimental aortic aneurysms. J Vasc Res 2009;47:355-66

19. Whittle IR, Dorsch NW, Besser M. Spontaneous thrombosis in giant intracranial aneurysms. J Neurol Neurosurg Psychiatry 1982;45:1040-47

20. Blanc R, Weill A, Piotin M, et al. Delayed stroke secondary to increasing mass effect after endovascular treatment of a giant aneurysm by parent vessel occlusion. AJNR Am J Neuroradiol 2001;22:1841-43

21. Strother C, Eldevik P, Kikuchi $Y$, et al. Thrombus formation and structure and the evolution of mass effect in intracranial aneurysms treated by balloon embolization: emphasis on MR findings. AJNR Am J Neuroradiol 1989;10: 787-96 